\title{
Comparing the fundamental limit of detection for interferometric and resonant biosensors with coherent phase read-out
}

\author{
Jonas Leuermann ${ }^{1,2}$, Íñigo Molina-Fernández ${ }^{1,2}$, Alejandro Ortega-Moñux ${ }^{1,2}$, \\ J. Gonzalo Wangüemert-Pérez ${ }^{1,2}$, and Robert Halir ${ }^{1,2}$ \\ 1. Bionand Center for Nanomedicine and Biotechnology, Parque Tecnológico de Andalucía, 29590 Málaga, Spain \\ 2. Universidad de Málaga, Dept. de Ingeniería de Comunicaciones, ETSI Telecomunicación, Campus de Teatinos s/n, 29071 Málaga, Spain
}

Integrated photonic biosensors are capable of detecting trace amounts of biochemical substances without timeconsuming labeling steps [1]. Ring resonators and Mach-Zehnder interferometers (MZI) are among the most widely used sensor architectures. The latter can operate with a simple, fixed-wavelength source and have recently shown state-of-the-art limit of detections (LOD) $\left(10^{-7}-10^{-8}\right.$ RIU) [2] by using the coherent phase read-out illustrated in Fig. 1(a), overcoming sensitivity fading and directional ambiguity [3]. Sensors using resonant structures often require a wavelength-swept source or broadband source combined with a spectrum analyzer [4]. However, they can also be interrogated with a coherent detection scheme and a fixed wavelength source, shown in Fig. 1(b), making them especially suited for cost sensitive point-of-care devices.

(a)

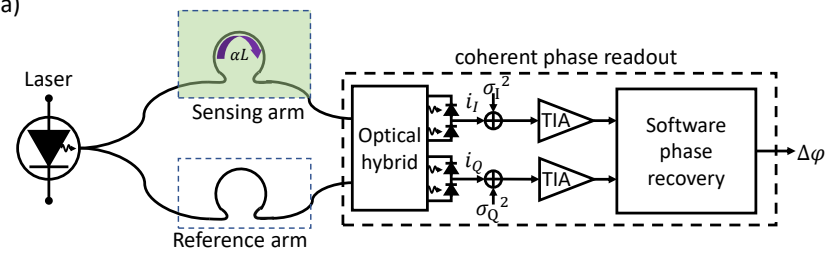

(b)

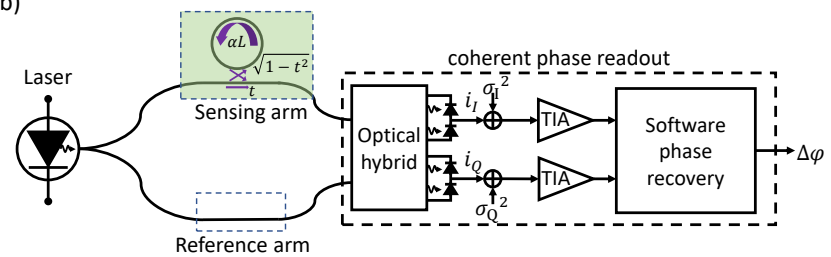

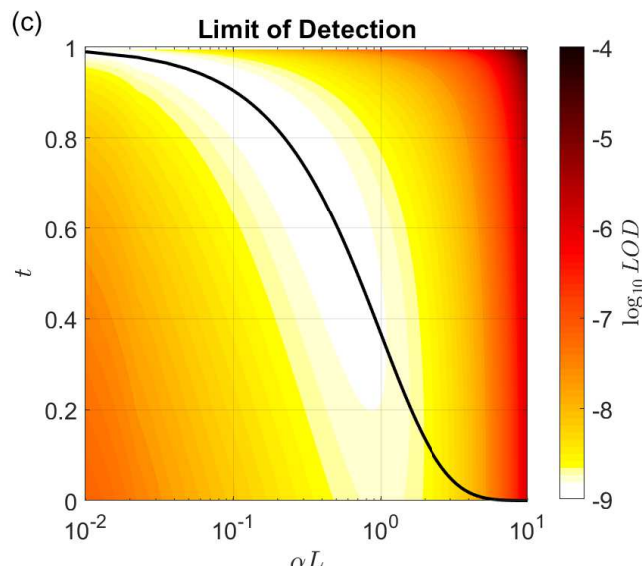

Fig. 1 Schematic of a biosensing system based on an interferometer (a) and a ring resonator (b) with coherent phase read-out. (c) LOD as a function of the absorption $\alpha L$ of the propagating light in the sensing arm and transmission coefficient $t$. The ring is assumed to be on resonance. The black curve corresponds to critical coupling, i.e. $t=\exp (-\alpha L)$. For $t=0$ the LOD results in the LOD of an interferometric sensing architecture.

The LOD is the smallest change in analyte that can be detected by the biosensing system. In addition to its dependency on the waveguide sensitivity, it is critically limited by the signal attenuation and noise [5]. We have analytically studied the impact of these factors on the LOD, arring at a closed form expression. This expression is valid for both interferometric and ring resonators with coherent detection scheme and holds for interferometric architectures with conventional read-out, at the quadrature point, too, thereby enabling us to formulate clear guidelines for sensor optimization [5]. Fig. 1(c) shows the LOD of the resonant structure depending on the transmission coefficient $t$ of the waveguide-ring coupler and its associated losses $\alpha L$ due to propagation. The LOD is obtained for a typical setup with SOI waveguides. Assuming the ring to be tuned to resonance it can clearly be observed that both structures have an optimum length $L=L_{\mathrm{opt}}$ for which the $L O D$ is minimized. For the resonant case the optimum length diminishes with increasing transmission coefficient $t>0$. We observe that for short sensor length $(\alpha L \ll 1)$, the optimum LOD is only achieved for a small range of transmission coefficients, thus requiring stringent control of fabrication tolerances. For the interferometric case, i.e. $t=0$, for which no tuning to resonance is required, the optimum lenght is $\sim 1 / \alpha$ reaching virtually the same LOD as a critically coupled ring resonator.

\section{References}

[1] A. F. Gavela, D. G. García, J. C. Ramirez and L. M. Lechuga, "Last advances in silicon-based optical biosensors,” Sensors, (2016), 16, 285. [2] R. J. J. van Gulik, B. M. de Boer, and P. J. Harmsma, "Refractive index sensing using a three-port interferometer and comparison with ring resonators," IEEE Journal of Selected Topics in Quantum Electronics, vol. 23, no. 2, pp. 433-439, (2017).

[3] R. Halir, L. Vivien, X. L. Roux, D.-X. Xu, and P. Cheben, "Direct and sensitive phase readout for integrated waveguide sensors," IEEE Photonics Journal, vol. 5, no. 4, p. 6800 906, (2013).

[4] L. Gounaridis et al., "High performance refractive index sensor based on low q-factor ring resonators and FFT processing of wavelength scanning data," Opt. Express 25, 7483 (2017)

[5] Í. Molina-Fernández, J. Leuermann, A. Ortega-Moñux, J. G. Wangüemert-Pérez, and R. Halir, "Fundamental limit of detection of photonic biosensors with coherent phase readout," Optics Express, submitted. 